\title{
Knowledge Evolution in Industrial Clusters
}

\author{
Langfeng Wang \\ School of Economic and Management, Southeast University \\ Nanjing 211189, China \\ E-mail: wanglangfeng@gmail.com \\ Qunhong Shen \\ School of Public Policy and Management, Tsinghua University \\ 1 Tsinghua Park, Beijing 100086, China \\ Tel: 86-10-6279-7178 E-mail: shenqh@tsinghua.edu.cn
}

This study is sponsored by NSFC (70102001)

\begin{abstract}
Character of knowledge management conducted in industrial clusters is different from entity of enterpresis and socail public deparment because of distinction in management objection. Many scholars was attracted to reseach knowledge management for enterprises, knowledge management in industrial clusters lack durable study in depth. This paper seeks to lay out an organizational foundation to a theory of industrial clusters and present the necessary, factors, process and characters of knowledge management in industrial clusters and can be as reference for policy-makers of industrial clusters.
\end{abstract}

Keywords: Knowledge management, Industrial clusters, Knowledge evolution

\section{Introduction}

It is widely observed that the society we live in has been gradually turning into a "knowledge society"(Drucher 1968; Bell 1973; Toffler 1990). The study of knowledge in itself is not a new topic. It extends back to the ancient philosophers. Its scientific study, however, can be traced back to 1950 s, when tremendous progress occurred in the cognitive sciences (Varun Grover and Thomas H. Davenport, 2001, pp 5-21). Though activity of knowledge management has experienced long history, theory system of knowledge management appeared in 1950s and formed its own theory systems at 1980s (Grover and Davenport, 2001). During the development of modern knowledge management, knowledge management has been applied by various social department and economic organization. Though many scholars have been researching knowledge management, few of them was devoted to knowledge management in industrial clusters.

Industrial clusters, as a special organization, are different from entity of enterprises and social public department. So knowledge management conducted in industrial clusters has its own characters. Many scholars was attracted to research knowledge management for enterprises, but knowledge management in industrial clusters lack durable study in depth. This paper presents the necessary, factors, process and characters of knowledge management in industrial clusters and can be as reference for Policy-makers of industrial clusters.

\section{Necessary of conducting knowledge management in industrial clusters}

Industrial clusters, as an important economic modality, have attracted attention of many scholars. Since notion of industrial clusters was presented by Porter, many country and district have been developing industrial clusters by plugging into substantial resources.

In worldwide scope, many industrial clusters have been appearing in traditional industry, such as the footwear industry in northern Italy, champagne in French, Ruhr industrial park in German, American Steel Strip, some district of Jiangsu, Zhejiang and Guangdong in China. At the same time, some industrial clusters appear in high technology which can representing the developing direction of new economic, such as Silicon Village in American, Boston 128 road, Cambridge Industrial Park in UK, Xinzhu district in Taiwan, Zhongguancun in Beijing, etc.

\subsection{Requirement of national Competition advantage and survival of enterprise}

National competitive advantage can be reflected by development level of industrial clusters of one country. With the passage of time, many industrial clusters disappeared, but other clusters become stronger and stronger and have been 
cultivating international firms with global competition. A very important reason for these competitive industrial clusters is their system foundation of communication and growing of knowledge elements which can improve the core capabilities of company, such as research and development capability, management and operation level, production management, etc., and furthermore promoting national economic growth. Knowledge evolution promotes enterprises development and intensify national competition advantage.

\subsection{Requirement for development of industrial clusters}

Porter proposed diamond model which can be used with industrial centralization theory to analysis national competitive advantage (1990) (Porter, 2002). The form and development of industrial clusters have many important factors, but system assurance for innovative network is an important reason. For spatial convenience, spillover and share of knowledge is an important reason for enhancing competitive advantage of enterprises in industrial clusters. A distinct character in the development period of industrial clusters is intensifying of communication and evolution function of knowledge elements from getting together of industrial factors to the foundation of mature knowledge network. In knowledge evolution, cooperation effect which has very important meaning for the development of high technology is fortified.

\section{Division of knowledge in industrial clusters}

Knowledge in industrial clusters can be divided into clusters knowledge, enterprises knowledge and personal knowledge by carrier, can also be divided into production management knowledge, production knowledge, market development knowledge, R\&D knowledge, management and operational knowledge. Every catalogues of knowledge has its own evolution path and transmission carrier.

By innovation knowledge in industrial clusters can be divided into tacit knowledge and explicit knowledge. Both types of knowledge are important. Explicit knowledge which can be easily codified has been expressed by logic system and language. The notion of tacit knowledge can be traced back to the philosopher Michael Polanyi, but has been applied to business and knowledge management by Japanese management scholar IKujiro Nonaka (Nonaka, I. and Takeuchi, H, 1995). Tacit knowledge which is embedded in the human brain cannot be expressed easily, and tacit knowledge belongs to environment, culture and preference etc which has support the innovation of knowledge but is difficulty to express by logic system and language. Much knowledge remains tacit for various resons. Perhaps its explication would not be beneficial. Unless an incentive is created, there is little reason for an individual or clusters possessing tacit knowledge that provides an important competitive advantage to explicate "away" that advantage.

Knowledge can be categorize into various form by various criteria (J. Zhang and Z. Liu, 2005). As shown in table 1.

Table 1. Various knowledge Category by Various Criteria

\begin{tabular}{l|l}
\hline Criteria & knowledge Category \\
source & Endogenous and Exogenous knowledge \\
Maturity & Regular and exceptional knowledge \\
Scope & Local and overall knowledge \\
State & Static and Dynamic Knowledge \\
Clarity & Explicit and Tacit Knowledge \\
Hierarchy & $\begin{array}{l}\text { Personal, Team and Organizational knowledge } \\
\text { Role }\end{array}$ \\
Fact-knowledge, Process-knowledge \\
Content & $\begin{array}{l}\text { control-knowledge and knowledge elements } \\
\text { fact, Principle, skills and Human knowledge }\end{array}$ \\
\hline
\end{tabular}

Economic modality is diversified by catalogues of knowledge in industrial clusters. Personal knowledge, enterprises knowledge and clusters knowledge have their own characters; tacit knowledge is different from dominant knowledge in characters. knowledge in clusters has mainly a form of network.

\section{Factors inflenting knowledge evolution in industrial ckusters}

Research of knowledge management in industrial clusters include exterior incentive, endogenetic variables, factors, conditions, propellent, platform and path which can promote practice activity of knowledge evolution in industrial clusters.

\subsection{System factors}

The creation of today's complex systems of products and services requires the merging of knowledge from diverse national, disciplinary, and personal skill-based perspectives. Innovation-whether it be revealed in new products and services, new process, or new organizational forms-is rarely an individual undertaking. Creative cooperation of 
knowledge elements is critical. To realize knowledge evolution in industrial clusters, connections among knowledge elements which accelerate knowledge evolution must exist. Because various form of knowledge elements (such as enterprises, persons) in industrial clusters and complex relations of competition and cooperation among them, institution protection and promotion should be designed more special to adapt for knowledge evolution and stable in industrial clusters. Definition of system factors has not been formed unified view (K P Sycara, 1998).

\subsection{Culture factors}

Culture foundation for centralization and communication of knowledge elements is a necessary and potential condition for knowledge evolution. Active culture promotes knowledge evolution in industrial clusters more strongly than conservative culture. Culture should be cultivated to coincide with the development of industrial clusters and cannot conflict with environment.

\subsection{Adaption for district and environment}

Knowledge evolution in industrial clusters must coincide with environment and meet with requirement of district economic development. Industrial clusters cultivate knowledge evolution and subjective choice system of industrial clusters may eliminate many production of knowledge evolution through selection or contest. Market of industrial clusters has many factors, such as consumer preference, economic and political environment, which can influence process of knowledge evolution strongly. Objective factors of district and environment bring opportunity and press for development of clusters and produce demand for knowledge evolution by culture, law and custom. And adaption for district and environment provide, at the same time, restrict the path, mode, method of knowledge evolution in a certain extent. It should be noted that the process of clusters knowledge evolution is a never-ending, circular process that is not confined to the environment. At the same time, the environment is a continual source of stimulation to knowledge evolution within clusters (Ikujiro Nonaka, 1994).

\subsection{Accumulation on knowledge elements and drive for knowledge element}

Knowledge evolution in industrial clusters needs accumulation of knowledge elements and adequate driving in a certain extent. Every step of knowledge evolution needs all factors engaging in it. In contrast with the knowledge evolution in enterprises and public department, knowledge elements has been cooperating and joining dynamically in knowledge evolution which are based on press of market and environment. Every evolution needs combination of various knowledge elements

\section{Process of knowledge evolution in industrial clusters}

The process of innovation is a rhythm of search and selection, exploration and synthesis, cycles of divergent thinking followed by convergence. Process of knowledge evolution in industrial clusters including 4 step: centralization of knowledge elements, network communication of knowledge elements, absorption of knowledge and achieving value of knowledge. These 4 steps constitute a knowledge evolution process.

\subsection{Centralization of knowledge elements}

The necessary condition of knowledge evolution in industrial clusters is firstly expressed by centralization of knowledge element. Industrial clusters are composed of enterprises, thus process of knowledge evolution was dynamically joined by knowledge elements. Techniques, skills, information and tactics for business operation has value for coping and diffuseness in a high certain to enterprises which have a high degree of similarity. As carriers of this technique and knowledge, engineers and managers turn into knowledge elements in industrial clusters. Centralization of knowledge elements which brings transmission, collision and innovation become the most important conditions in the nature of things.

\subsection{Network for communication of knowledge elements}

Communication of knowledge elements provides network connection. It is by virtue of such links that knowledge communication goes along in large-scale and knowledge innovation progress in multidimensional. Competition in industrial clusters weakens cooperation of knowledge elements, so policy-makers of clusters should intensify communication network of knowledge elements to accelerate the step of knowledge evolution. It is the best development form for knowledge evolution in clusters that mechanism protection for network of communication in knowledge elements finalizes by law.

By contrast with conceptions of groups as bounded entities within an clusters, evolving communication communities of practice are "more fluid and interpenetrative than bounded, often crossing the restrictive boundaries of the organization to incorporate people from outsides"(Brown and Duguid 1991, p.49). Moreover, these communities for communication can provide important contributions to visions for future development. Thus these communities communication represent a key dimension to socialization and its input to the overall knowledge creation process (Ikujiro Nonaka, 1994). 


\subsection{The absorption of knowledge}

The absorption of knowledge including two aspects: absorption of environment and absorption by persons. Absorption of environment mainly including integration knowledge elements with local customs, culture and economics entity, and absorption by persons is that knowledge element become a necessary department of personal capacity structure. Only by coping and transmission various knowledge elements, practice and theory evolution of knowledge advance greatly. Practice and theory of knowledge evolution which is based on the absorption of knowledge elements in enterprises and department progress greatly. of environment mainly including tacit knowledge. Absorption involves the conversion of explicit knowledge into more complex sets of explicit knowledge. In this stage, the key issues are communication and diffusion processes and the systemization of knowledge.

\subsection{Achieving the value of knowledge}

An important motivity for knowledge elements in industrial clusters is acquiring market value. It is by acquiring market value that knowledge elements strive for innovation and evolution. Value realization attracts centralization of knowledge element, and thus form a new cycle of knowledge evolution. This is a process of continuously strengthen cycle.

Four important steps constitute a cycle of knowledge evolution, as shown in figure 1.

Many cycle knowledge evolution in turn. Knowledge evolutionary process which is composed by many cycle of thus components show a spiral rose process, as shown in figure 2.

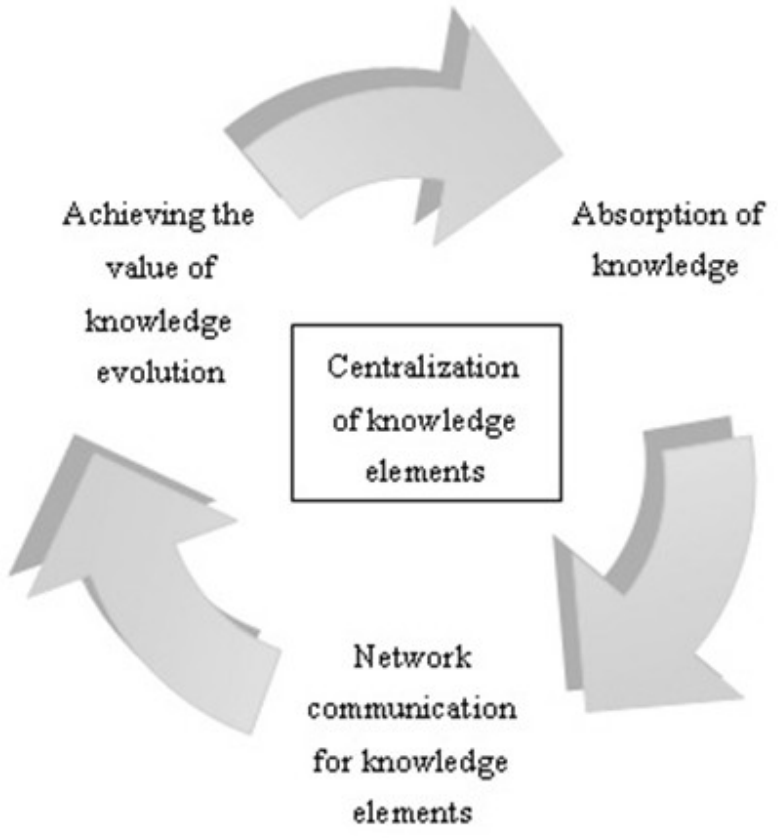

Figure 1. Process of knowledge evolution

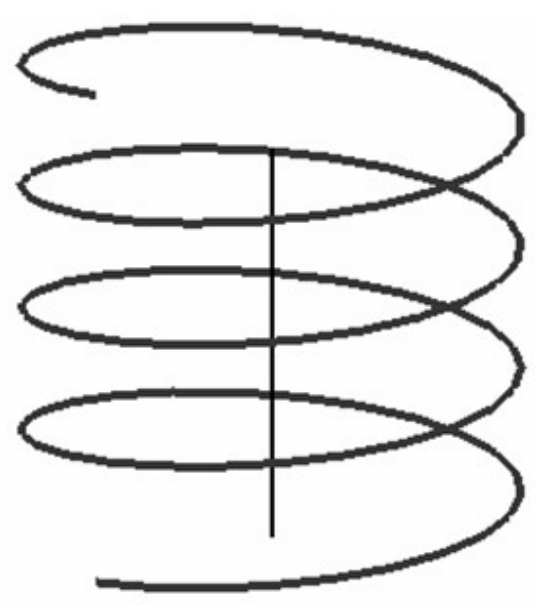

Figure 2. Spiral path of knowledge evolutions in clusters

\section{New charaters of knowledge evolution in industrial clusters}

Knowledge evolution in industrial clusters shows new characters which is different from various entities of enterprises and public department. Communication and extraction of information is important especially for opening system for enterprises in clusters. Skill cooperation, communication of innovative views and thinks of dominant and tacit knowledge transmission and evolution of knowledge in mature industrial clusters intensify knowledge evolution which intensifying competition of enterprises in turn.

\subsection{Transmission in network}

Knowledge transmission in industrial clusters is a process of forming solid network which need every elements participating in it and form effect communication mechanism. This solid network construction must be under institutional protection which is assurance for connections of knowledge elements. Knowledge elements produce effect of interaction effect and then realizing knowledge evolution by this communication network.

\subsection{Dynamic evolution}

Knowledge elements dynamically joined evolution in industrial clusters and changes with the environment and by value. Solid network for connections of knowledge elements changes dynamically. Centralizations and lost of knowledge elements, relations for connections of knowledge elements, path and breakthrough points of knowledge evolution 
changes dynamically with time and environment. Knowledge evolution prosperous in industrial clusters just because of dynamic properties of knowledge elements.

\subsection{Value-oriented}

Knowledge evolution is value-oriented, market profit stimulate a new cycle of knowledge evolution. Meeting marketing demand is the most important driving power for knowledge evolution. Meeting demand of market is important and as firsthand driving for knowledge evolution for enterprise. The same is true for knowledge evolution in industrial clusters. Though the entity of knowledge evolution in industrial clusters is fuzzy in a certain extent, knowledge evolution in industrial clusters has its own direction and path for evolution which is decided by elements joining in knowledge evolution synthetically.

\subsection{System evolution}

Knowledge evolution in industrial clusters is not tamper with single factors and conditions, but influenced by synthetically power. Knowledge evolution in industrial clusters mainly includes tacit knowledge such as knowledge accumulation, knowledge environment, institution of knowledge evolution which is different from enterprises and public department. Tacit knowledge and factors affecting it changes silently. Factors of dominant and tacit knowledge play roles in knowledge evolution together.

\subsection{Multidimensional direction for evolution}

Knowledge evolves in multidimensional in industrial clusters in that knowledge elements share culture, environmental, system and spillovers effect of knowledge. For market value every knowledge elements evolve in the various direction. So in the level of industrial clusters knowledge evolution happens in different department and in various directions.

\section{Conclusion and advices for policy}

\subsection{Construction platform for knowledge element communication}

Industrial clusters have its own characters which are different from concrete economic morphology. Policy-makers of industrial clusters should emphasis platform construction of knowledge evolution. Knowledge evolution intensifies competitive advantages for the long development of industrial clusters. A good platform draws amounts of knowledge elements and boost communication of knowledge elements. The platform should assure of culture innovative atmosphere which actitive knowledge elements to join in knowledge evolution.

\subsection{Institutional protection for knowledge evolution}

Only by institution assurance for knowledge evolution do knowledge evolution progress. Market value and communication network should be emphasized by institutional construction by which knowledge evolution progress greatly. Relation of cooperation and competition for knowledge elements joining in evolution is fuzzy and dynamic, so implementation of a institution protection accord with of the individual characteristics.

\subsection{Professional judgment for knowledge development}

Every industrial clusters have its own target market, every enterprises in industrial clusters has its own location of market and joined in process of knowledge evolution dynamically. More categories of knowledge in industrial clusters evolve in multidimensional. Direction of knowledge evolution must be choosed by professional judgment of policy makers. Policy makers and promoters for industrial clusters must have professional knowledge and experience in knowledge management.

\section{References}

Varun Grover and Thomas H. Davenport. (2001). General Perspectives On Knowledge Management: Fostering a Research Agenda. Journal of Management Systems summer. Vol 18, No 1, pp 5-21.

Porter. (2002). National competitive advantage. Beijing: Huaxia Press.

Nonaka, I. and Takeuchi, H. (1995). The knowledge-creating company-How Japanese Companies Create the Dynamics of Innovation. Oxford: Oxford University Press.

J. Zhang and Z. Liu.(2005). Analysis on system factors in knowledge management. SCIENTIFIC MANAGEMENT RESEARCH, Vol. 23 No.2 Apr.

K P Sycara. (1998). Multi-Agent systems. AI Magazine, 19 (2):79-92.

Ikujiro Nonaka. (1994). A Dynamic Theory of Organizational Knowledge Creation. Organization Science, Vol.5, No.1. 